\title{
Renal biopsy: still a game changer
}

\author{
Catarina Abrantes ${ }^{1, *}$ (D), Patrícia Valério ${ }^{1, *}$ (iD), Teresa Furtado $^{1}$ (iD), Elsa Soares ${ }^{1}$ (iD, Patrícia Domingues $^{1}$ (iD, \\ Lúcia Parreira $^{1}$ (D), Mário Góis ${ }^{2,3}$ (iD), Helena Sousa ${ }^{2,3}$ (iD \\ * Equally contributing first authors \\ ${ }^{1}$ Nephrology Department, Setubal Hospital Center, Setubal, Portugal \\ ${ }^{2}$ Nephrology Department, Hospital Curry Cabral, Central Lisbon University Hospital Center, Lisboa, Portugal \\ ${ }^{3}$ Laboratory of Renal Morphology, Nephrology Department, Hospital Curry Cabral, Central Lisbon University Hospital Center, Lisboa, Portugal
}

\section{CLINICAL PRESENTATION}

We report the case of a melanodermic 55-year-old male patient admitted to the emergency department with headache and vomiting for less than 24 hours. He had no known kidney disfunction or other medical history. On admission he was hypertensive $(215 / 116 \mathrm{mmHg})$, with no other relevant findings on physical examination. Bloodwork showed anemia (hemoglobin $9.8 \mathrm{~g} / \mathrm{dL}$ ), kidney injury (creatinine $7.53 \mathrm{mg} / \mathrm{dL}$, urea $170 \mathrm{mg} / \mathrm{dL}$ ), metabolic acidosis and hyperkalemia. Urinalysis revealed occasional proteinuria $70 \mathrm{mg} / \mathrm{dL}$ (urine protein to creatinine ratio $0.7 \mathrm{~g} / \mathrm{g}$ ) and erythrocyturia of $154 \mathrm{u} / \mathrm{L}$. Renal ultrasound revealed symmetrical normal-sized kidneys with normal corticomedullary differentiation. Further laboratory tests revealed: 175.000/uL platelets, albumin 3g/L, LDH 284U/L (without haptoglobin reduction or schistocytes on smear blood test), fasting blood glucose $98 \mathrm{mg} / \mathrm{dL}$, hemoglobin A1c 5.3\%, negative serologic tests for $B$ and $C$ hepatitis and HIV infection, normal serum protein electrophoresis, normal serum free kappa and lambda chains without abnormal ratio, normal C3 and C4 levels and negative immunological panel including ANA, ANCA and anti-GBM. He also underwent transthoracic echocardiography and fundoscopy examination, which documented concentric hypertrophy and grade 2 hypertensive retinopathy, respectively.

The patient's kidney function continued to deteriorate, even though urine output was preserved and intermittent hemodialysis was initiated. A kidney biopsy was therefore performed.

\section{QUESTIONS}

1. What is the most likely diagnosis?

2. Considering the kidney biopsy results, what is the final diagnosis?

3. What treatment options can we offer to the patient?

\section{ANSWERS}

\section{What is the most likely diagnosis?}

Our patient presented with renal failure and severe hypertension. Since analytic investigation was inconclusive and given the findings of hypertensive retinopathy and concentric hypertrophy, our first hypothesis was malignant hypertension. This entity is frequently complicated by renal dysfunction and in a subset of affected patients it presents as oliguric renal failure or rapidly progressive renal failure, requiring kidney replacement therapy during the acute phase ${ }^{1}$. We expected to find typical acute ischemic changes secondary to medial hypertrophy, intimal hyperplasia and fibrinoid necrosis of small arteries and arterioles on renal biopsy.

\section{Considering the kidney biopsy results, what is the final diagnosis?}

The kidney biopsy specimen showed renal cortex containing 13 glomeruli and medium-sized arteries. Under light microscopy, 6 glomeruli with hyalinosis/sclerosis were observed, and the remaining ones presented mesangial proliferation, with nodular formations and thickening and duplication of Bowman capsules (Figure 1 and 2). Interstitial fibrosis and tubular atrophy covered around $75 \%$ of fragment, while tubules showed severe thickening and duplication of tubular basement membranes in both atrophic and non-atrophic tubules (Figure 3). Arterioles presented severe hyalinosis and small and medium-sized arteries showed severe intimal thickening (Figure 1). Congo red stain was negative (Figure 4). In the immunofluorescence study, linear deposition of albumin and IgG were observed (Figure 5). These histopathological findings support the diagnosis of diabetic nephropathy (DN) with nodular sclerosis (stage III). However, our patient had no history of diabetes, hemoglobin A1c was 5.3\%, fasting glucose was normal, and capillary blood glucose levels, measured two to three times daily, were within the normal range. 


\section{Figure 1}

Periodic acid-Schiff (PAS) stain, magnification x400: Mesangial matrix proliferation, forming PAS-positive Kimmelstiel-Wilson nodules (arrow); arterioles show severe hyalinosis $(*)$.

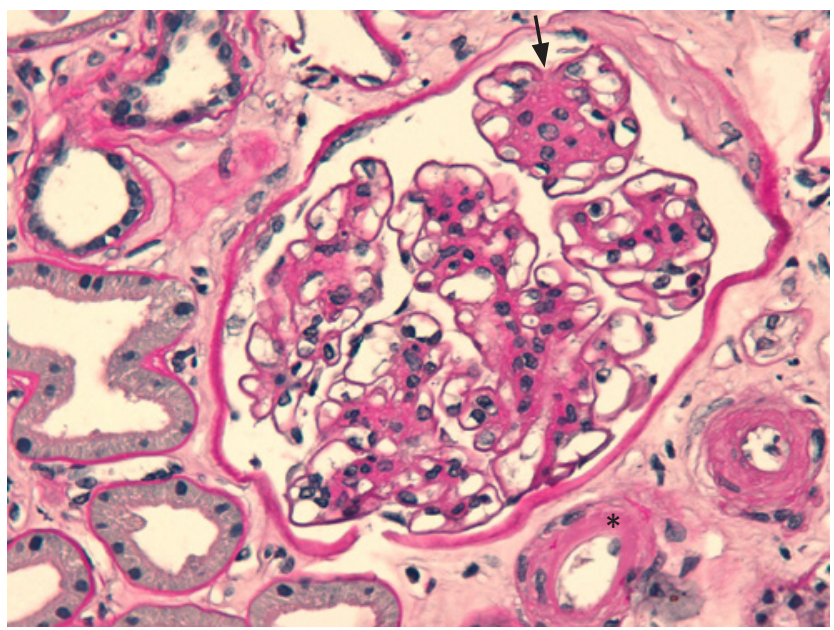

\section{Figure 2}

PAS stain, magnification x400: capillary obliteration by hyalin and sclerotic lesions, suggesting recurrent episodes of injury.

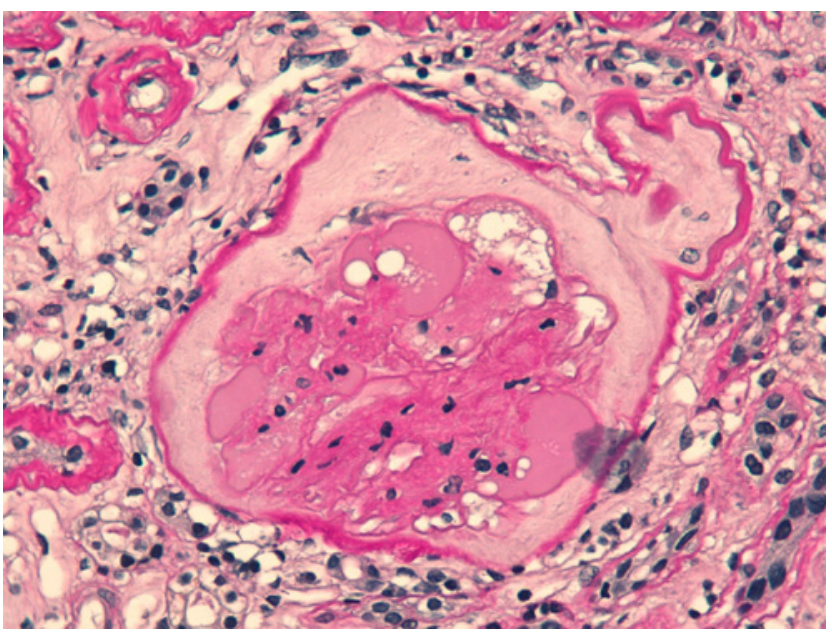

Several conditions can cause a nodular glomerulosclerosis pattern, mostly distinguishable through immunofluorescence or electron microscopy $^{2}$. Dysproteinemias, such as amyloidosis and deposition disease were excluded with Congo red stain and immunofluorescence, respectively. However, despite immunofluorescence being negative, an evaluation on deparaffinized tissue should be performed to guarantee a true negative result. Other deposition diseases, such as fibrillary and immunotactoid glomerulonephritis, can only be truly excluded with electron microscopy, despite light microscopy and immunofluorescence features not being suggestive. Idiopathic nodular glomerulosclerosis is a diagnosis

\section{Figure 3}

Masson's trichrome stain, magnification $\times 100$ : sclerotic glomerulus $(*)$ and another one with Kimmelstiel-Wilson nodule (arrow); between the glomeruli there is an area of interstitial and tubular fibrosis, with inflammatory infiltrate within atrophic areas.

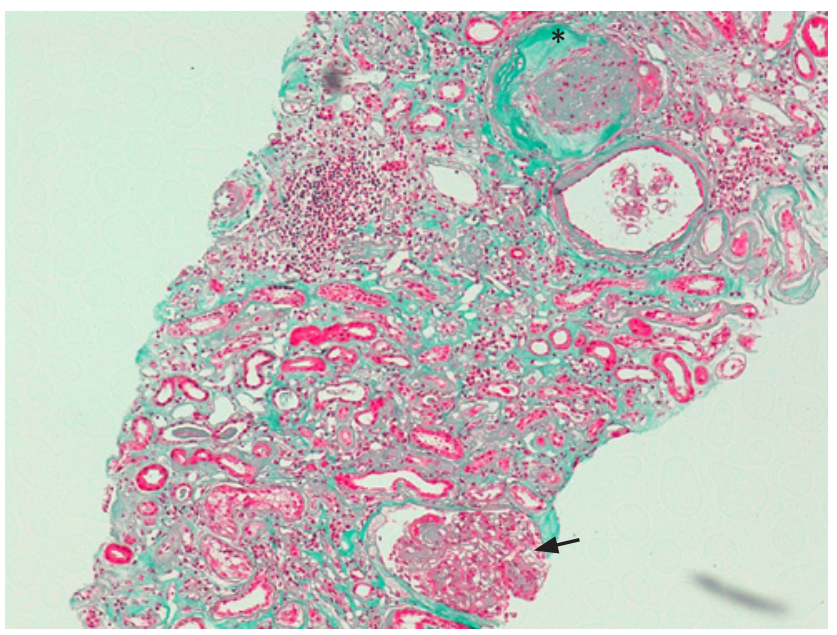

\section{Figure 4}

Congo red stain, magnification $\times 400$ : nodules are Congo red-negative.

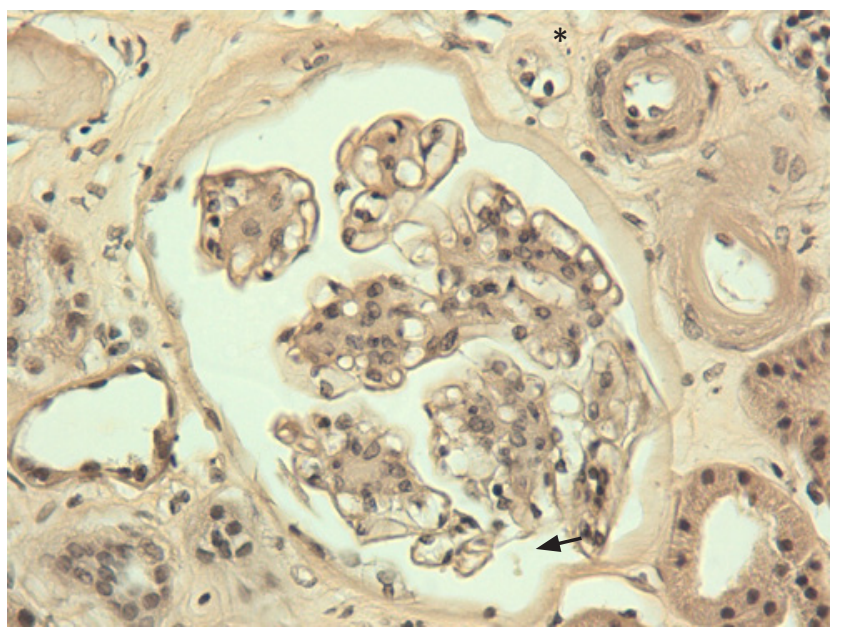

of exclusion, frequently associated with smoking and hypertension. The patient in this case was a non-smoker.

Increasing reports have documented nodular glomerulosclerosis without any manifestation of diabetes ${ }^{3-6}$. In a review by López-Revuelta et $\mathrm{al}^{7}$, a total of 95 cases of patients with DN without diabetes (DNWD) were reported between 1994 and 2015. According to the available literature, DNWD is linked to hypertension and other recognized cardiovascular risk factors, including overweight or metabolic syndrome. Indeed, our patient presented with uncontrolled hypertension and 


\section{Figure 5}

Immunofluorescence - albumin, magnification x400.

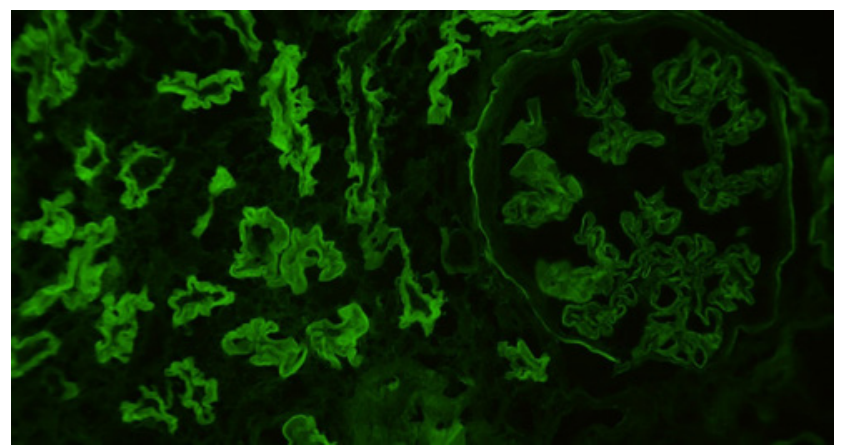

suffered from overweight. The underlying pathophysiology is not yet understood and controversy remains as to whether this rare entity is actually part of idiopathic nodular glomerulosclerosis or if an increased sensitivity to a non-diabetic range of blood glucose levels could result in an exaggerated glomerulovascular response among those affected ${ }^{8}$.

After this surprising result, an oral glucose tolerance test was performed, confirming the diagnosis of diabetes (fasting blood glucose: $98 \mathrm{mg} / \mathrm{dL}$; blood glucose level after 2 hours: $211 \mathrm{mg} / \mathrm{dL}$ ).

In conclusion, this case report should alert the nephrology community to a possible new disease entity in patients with several cardiovascular risk factors, such as impaired glucose metabolism, hypertension, metabolic syndrome, obesity and hyperlipidemia. This histologic pattern presents with alterations in glomeruli and tubules (both in morphologic and immunofluorescence studies) compatible with diabetic nephropathy in patients without diabetes or with late-onset diabetes. The abovementioned cardiovascular risk factors are possibly linked to metabolic abnormalities that cause changes in glomerular blood flow regulation with hyperfiltration and ischemia that, in a susceptible genetic background, can cause this renal histologic pattern.

\section{What treatment options can we offer to the patient?}

Management of DN is directed towards optimal glycemic control and strategies to reduce the risk of kidney disease progression and cardiovascular disease.
Unfortunately, in the present case, renal recovery didn't occur and the patient maintained dialysis dependence. In this scenario, without evidence of other end organ injury, treatment options are limited to the control of cardiovascular risk factors.

Disclosure of potential conflicts of interest: none declared.

\section{References}

1. Amraoui F, Bos S, Vogt L, et al. Long-term renal outcome in patients with malignant hypertension: a retrospective cohort study. BMC Nephrology 2012; 30;13:71.

2. Najafian B, Fogo AB, Lusco MA, et Al. Atlas of renal pathology: diabetic nephropathy. Am J Kidney Dis 2015;66(5):e37

3. Sanai T, Okuda S, Yoshimitsu T, et al. Nodular glomerulosclerosis in patients without any manifestation of diabetes mellitus. Nephrology 2007;12:69-73

4. Chang CS, Yang AH, Chang CH. Nodular glomerulosclerosis mimicking diabetic nephropathy without overt diabetes mellitus. Clin Nephrol 2005;64:300-304

5. Kusaba T, Hatta T, Sonomura K, et al. Idiopathic nodular glomerulosclerosis: three Japanese cases and review of the literature. Clin Nephrol 2007;67:32-37

6. Wu J, Yu S, Tejwani V, et al. Idiopathic nodular glomerulosclerosis in chinese patients: a clinicopathologic study of 20 cases. Clin Exp Nephrol 2014;18:865-875

7. López-Revuelta K, Abreu A, Gerrero-Márquez C, et al. Diabetic nephropathy without diabetes. J Clin Med 2015;4(7):1403-1427

8. Kuppachi S, Idris N, Chander PN, et al. Idiopathic nodular glomerulosclerosis in a non-diabetic hypertensive smoker-case report and review of literature. Nephrol Dial Transplant 2006;21:35713575

\section{ORCID}

Catarina Abrantes (iD) 0000-0002-1093-3395

Patrícia Valério iD 0000-0001-7397-0233

Teresa Furtado iD 0000-0002-0714-0060

Elsa Soares iD 0000-0002-6748-6063

Patrícia Domingues iD 0000-0001-5172-2917

Lúcia Parreira iD 0000-0003-1235-2567

Mário Góis (iD) 0000-0002-0937-8611

Helena Sousa iD 0000-0002-7644-2230

\section{Correspondence to:}

Catarina Abrantes, MD

E-mail: catarina.g.abrantes@gmail.com

Patrícia Valério, MD

E-mail: p.valerios89@gmail.com 